\title{
TRANSFER FUNCTION PARAMETERS ESTIMATION BY SYMMETRIC SEND-ON-DELTA SAMPLING
}

\author{
José Sánchez, María Guinaldo, Sebastián Dormido \\ Departamento de Informática y Automática, UNED, C/ Juan del Rosal 16, 28040 Madrid, \\ \{jsanchez,mguinaldo,sdormido\}@dia.uned.es
}

Antonio Visioli

Department of Mechanical and Industrial Engineering, University of Brescia, via Branze 38, 25123 Brescia, antonio.visioli@unibs.it

\section{Resumen}

In this paper, a method to estimate the parameters of first and second order systems with time delays with different accuracy levels for autotuning of event-based PID controllers is presented. Information from the describing function of the nonlinearity that introduces the event-based sampling strategy and measures of the oscillations are used to generate the estimations. The event-based sampling condition applied is based on the sampling strategy known as symmetric-send-on-delta.

Palabras clave: Describing function, eventbased control, send-on-delta, estimation, limit cycle.

\section{INTRODUCTION}

During last years, studies on the application of the event-based paradigm to different control engineering fields have experimented a huge expansion both in printed publications and in conferences and workshops. From the first two publications in an international conference with an explicit mention to event-based control [1] and sampling [2] to these days, the event-based paradigm has been applied to PID control, predictive control, networked control, distributed control, agentbased systems, filtering, state estimation, sampling, etc. (see [11]). However, there is one specific field in control engineering in which the eventbased paradigm has not been applied until now: parameter estimation of transfer functions. To find references on system identification that can be considered near or related to the event-based paradigm, it is necessary to look at works on autotuning based on relays, such as $[8,10,13,15]$.

As a relay is a static non-linearity, the common procedure in the frequency domain to analyze the behavior of a relay in a feedback loop is the describing function (DF). This method relies on the estimation of the amplitude $\left(K_{u}\right)$ and frequency $\left(\omega_{u}\right)$ of the oscillations produced by a relay, which are approximations to the true values due to the truncated high order harmonics that the DF ap- proach introduces even with no measurement errors.

The relationship between the describing function approach and the parameter estimation based on events is due to the fact that an event-based sampling can generate an oscillatory signal in the control loop. In the most basic architecture of an event-based feedback control loop, the sampling is done applying a Send-On-Delta (SOD) sampling strategy to the process output $[12,14]$. When the output is quantified by a quantity multiple of $\delta$, the relationship between the input and the output of the event sampling block is symmetric with respect to the origin. Then, this strategy is known as Symmetric-Send-On-Delta (SSOD) and can be seen also as a generalization of a biased relay [3] with its corresponding describing function.

The present paper provides an event-based parameter estimation approach for autotuning purposes of control loops using a SSOD block located at the process output. A first try on using the describing function approach to autotuning of eventbased PI control loops was reported in [4]. In this work, the process (first order plus time delay, FOPTD for short) parameters are estimated considering a limit cycle of one level generated by the SSOD block and knowing at first one of the three FOPTD parameters. Also, the parameter estimation in an event-based control loop is considered in [5]. In such work, the estimation approach is based on curving fitting using a relay in series with the SSOD block and a pre-tuned PI controller. From measurements obtained of the oscillatory signals and using the controller parameter values, expressions to obtain the FOPDT parameters are given. In the new approach presented here, a previous tuning of the controller is not necessary and the method can be applied at the beginning of the design phase, as a previous step to the tuning. Like previous works based on DF, the model parameters need to be calculated using some previous information from the process (i.e., the static gain or the velocity gain in a process with integration), but no additional experiments are needed.

The rest of the paper is organized as follows. In Section 2, the structure of the control loop con- 


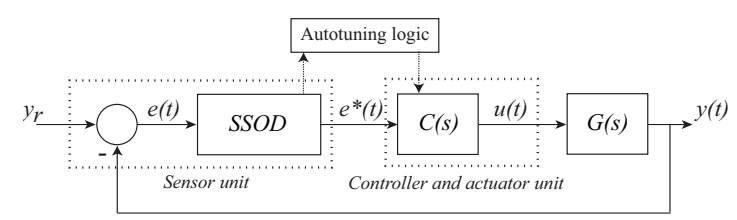

Figure 1: Event-based control loop where the estimation parameter approach is included as part of the autotuning logic.

sidered is described. In Section 3, the estimation approach based on the describing function of the SSOD block is presented. Section 4 outlines the procedure to identify the most usual models used for tuning of PID controllers. Examples of results are shown in Section 5. Finally, the discussion about the goodness of the proposed method is given in Section 6 .

\section{ARCHITECTURE}

The event-based feedback control loop considered in this work is divided into three units (Figure 1): the sensor, the control, and the actuator. The sensor unit is composed of the sensor and its on-board intelligence, and it is on charge of computing the error $e(t)=y_{r}(t)-y(t)$. The event-based sampler takes the error $e(t)$ and produces an eventbased signal $e^{*}(t)$ transmitted to the controller. The control unit $C(s)$ implements the control law that, without loss of generality, is considered to be PID. The actuator unit receives $u(t)$ from $C(s)$ and applies it to the process $G(s)$.

The autotuning logic block contains the programming code needed to apply the different steps of the estimation approach explained in this paper. So, during the identification phase, the logic would be in charge of deactivating the integral and derivative actions of the PID in Figure 1 and modify the proportional gain to produce the oscillations needed to estimate the parameters. At the same time, the logic would introduce changes in the reference $y_{r}$ to disturb the process (for example, a step or a pulse). Once the event-based estimation phase concludes, the $C(s)$ would start working with the information received from the autotuning logic, being these data, for example, the three PID parameters derived from a set of tuning rules or directly the model parameters for the predictive controller.

In this paper, the event-based sampling is done applying a SSOD. Formally, a SOD sampler can be thought as a block which receives a continuous signal $e(t)$ as input and generates a sampled signal $e^{*}(t)$ as output, which is a piecewise constant signal with $e^{*}(t)=e\left(t_{k}\right), \forall t \in\left[t_{k}, t_{k+1}\right)$. Each $t_{k}$ is denoted as event time, and it holds

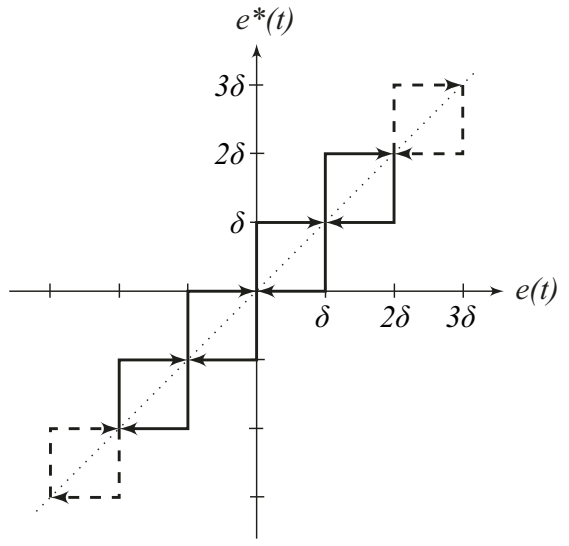

Figure 2: Relationship between $e(t)$ and $e^{*}(t)$.

that $t_{k+1}=\inf \left\{t\left|t>t_{k},\right| e(t)-e\left(t_{k}\right) \mid \geq \delta\right\}$, where $\delta>0$ is the sampling threshold. In the symmetric case (SSOD), the sampled output signal $e^{*}(t)$ can assume only values multiple of the threshold $\delta$, and is mathematically described as follows:

$e^{*}(t)= \begin{cases}(i+1) \delta & \text { if } e(t) \geq(i+1) \delta \wedge e^{*}\left(t^{-}\right)=i \delta \\ i \delta & \text { if } e(t) \in[(i-1),(i+1)] \delta \wedge e^{*}\left(t^{-}\right)=i \delta \\ (i-1) \delta & \text { if } e(t) \leq(i-1) \delta \wedge e^{*}\left(t^{-}\right)=i \delta\end{cases}$

The key point of the relationship between $e(t)$ and $e^{*}(t)$ is that it can be considered as a generalization of a relay with hysteresis, where there is an infinite number of thresholds [9], as shown in Figure 2. This implies that a describing function of this non-linearity can be derived and applied to the analysis of the oscillations.

\section{THE DESCRIBING FUNCTION APPROACH}

The describing function of a SSOD block that produces a signal similar to Figure 2 is given by the following equation $[6,7]$ :

$$
\begin{aligned}
N(A, \delta)= & \frac{2 \delta}{\pi A}\left[1+\sqrt{1-\left(\frac{\delta m}{A}\right)^{2}}+2 \sum_{k=1}^{m-1} \sqrt{1-\left(\frac{\delta k}{A}\right)^{2}}\right] \\
& -j \frac{2}{\pi}\left(\frac{\delta}{A}\right)^{2} m
\end{aligned}
$$

considering that the input is a sinusoidal signal of amplitude $A$. In (1), $m=$ floor $(A / \delta)$ represents the amplitude of the oscillation reached by the signal $e(t)$. According to Figure 2, the output of the SSOD block is zero for inputs of amplitude $A<\delta$, so $A$ must be equal or higher than $\delta$ in order to produce some output. For this reason, the quotient $\delta / A \in[0,1]$ and can be considered as a normalized parameter $\delta_{A}$. So, the DF expression of the SSOD block can be rewritten as

$$
\begin{aligned}
N\left(\delta_{A}\right)= & \frac{2 \delta_{A}}{\pi}\left[1+\sqrt{1-\left(\delta_{A} m\right)^{2}}+2 \sum_{k=1}^{m-1} \sqrt{1-\left(\delta_{A} k\right)^{2}}\right] \\
& -j \frac{2 \delta_{A}^{2}}{\pi} m,
\end{aligned}
$$




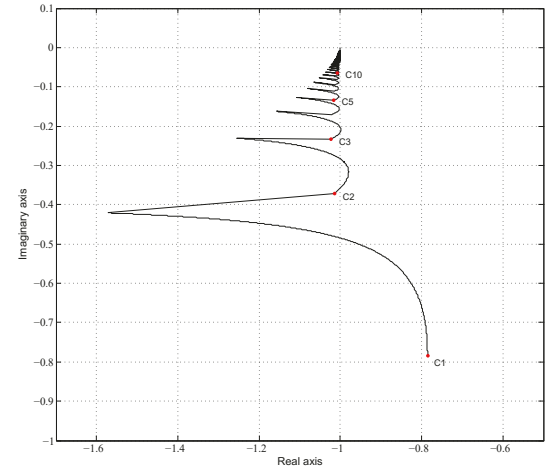

Figure 3: Nyquist plot of $-1 / N\left(\delta_{A}\right)$. A $C_{i}$ point corresponds to $\delta_{A}=1 / i$.

where $\delta_{A} \in[0,1]$. The calculations of $N(A, \delta)$ are omitted for brevity.

The condition for the existence of limit cycles is given by [33]:

$$
G_{o l}(j \omega)=-\frac{1}{N \delta_{A}},
$$

where $G_{o l}(j \omega)=C(j \omega) G(j \omega)$. In Figure 3, the portrait of $-1 / N\left(\delta_{A}\right)$ is represented for $\delta_{A} \in[0,1]$ and some particular points are illustrated. The intersection of $G_{o l}(j \omega)$ with a point $C$ of $-1 / N\left(\delta_{A}\right)$ is known as intersection or critical point. The subindex $i$ in a $C$ point means that its intersection with $G_{o l}(j \omega)$ produces theoretically an oscillation of amplitude $A=i \delta$, $i \geq 1$. As an oscillation of amplitude $A=i \delta$ with $i=1, \ldots, n$ means that $m=[A / \delta]=[i \delta / \delta]=i$, from (2) the expression to obtain the $C_{i}$ corresponding to an oscillation of amplitude $i \delta$ is

$$
C_{i}=\frac{-1}{\frac{2}{i \pi}\left[1+2 \sum_{k=1}^{i-1} \sqrt{\left.1-\left(\frac{k}{i}\right)^{2}\right]}-j \frac{2}{i \pi}\right.} .
$$

In Figure 4.a, each arc segment of the SSOD represents a certain type of limit cycle. The first arc, starting in $C_{1}$, represents possible limit cycles of amplitudes in the interval $[\delta, 2 \delta)$ and corresponds to the triggering of events at crossing the $\pm \delta$ thresholds (Figure 4.b), the second arc represents possible limit cycles of amplitudes $[2 \delta, 3 \delta)$ generated at crossing the $\pm 2 \delta$ thresholds (Figure 4.c), and so on (Figure 4.d). Two or more intersection in the Nyquist map means that the process can reach two or more oscillations of different frequencies and amplitudes depending on the operation conditions. It is important to notice that $N\left(\delta_{A}=0\right)=C_{\infty}=\lim _{t \rightarrow \infty} C_{i}=-1$, that means the intersection of $G_{o l}(j \omega)$ with $C_{\infty}$ would produce a limit cycle of infinite amplitude, i.e., instability.

Applying a proportional gain $C(j \omega)=K_{\text {osc }}$ to a stable $G(j \omega)$, three possible cases can be generated:

- Case 1: $G_{o l}(j \omega)<-1$ and $G(j \omega)<-1 / N\left(\delta_{A}\right)$. No oscillations at any frequency.

- Case 2: $G_{o l}(j \omega)<-1$ and $G(j \omega)=-1 / N\left(\delta_{A}\right)$. Existence of oscillations at frequency $\omega_{o s c}$ and amplitude given by the intersection point, that

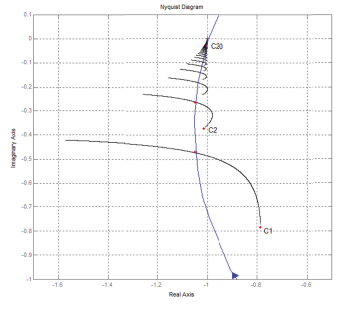

(a)

(d)

(b)

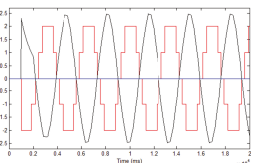

(c)

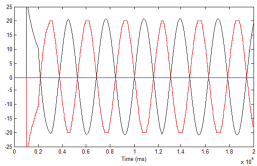

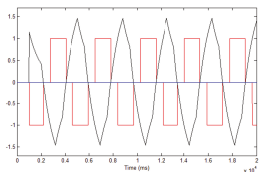

Figure 4: Graphical determination of the limit cycles of $G_{o l}(s)=2.25 e^{-s} /(s+1)$.

is, $A=\left[1 / \delta_{A}\right]$. There can be as many stable limit cycles as intersections.

- Case 3: $G_{o l}(j \omega) \geq-1$. Instability. However, depending of the operation or initial conditions of the process, the stable limit cycle can be reached avoiding the instability. Hence, for small perturbations, as the limit cycle is locally stable, it will converge to its original state with increasing time, avoiding the instability.

The event-based method for parameter estimation consists in applying a proportional gain $K_{o s c}$ to the unknown process $G(s)$ and forcing it to enter in a limit cycle of amplitude $i \delta, i \geq 1$. That means to move the process from Case 1 to Cases 2 or 3 . The details of the approach will be given in the next section, but a further comment is required before. The value of $C$ from the DF analysis is an approximation of the true value of the experimental oscillations denoted as $C^{\prime}$. Higher accuracy in the estimation depands higher oscillations, but, at the same time, the sensibility of $K_{\text {osc }}$ is higher.

\section{THE PARAMETER ESTIMATION PRCEDURE}

The transfer functions models used for PID autotuning and the equations derived for them are summarized in Table 1. We make the following assumptions: (i) the process is in steady state with the SSOD block centered in zero; (2) $K_{\text {osc }}$ is set to 1 .

The estimation procedure can be divided into the following steps:

1. Getting the oscillation parameters.

(a) To reach an stable oscillation of high order, i.e. $i>5$, move out the process from the steady state introducing a square pulse of amplitude, for example, $y_{r}=y_{\max _{r}}$ and increasing the $K_{\text {osc }}$ gain.

(b) Once the oscillation is stable, annotate the gain $K_{o s c}$, the amplitude of the oscillation 
Table 1: Models used for PID autotuning. $K, L, T, T_{1}$ and $T_{2}$ are all positive constants.

\begin{tabular}{|c|c|c|c|}
\hline Model & $\mathbf{G}(\mathbf{s})$ & $|\mathbf{G}(\mathbf{s})|$ & $\angle \mathbf{G}(\mathbf{s})$ \\
\hline FOPTD & $\frac{K e^{-L s}}{T s+1}$ & $\frac{K}{\sqrt{1+\omega^{2} T^{2}}}$ & $-\omega L-\arctan \omega T$ \\
\hline SOPTD-1 & $\frac{K e^{-L s}}{(T s+1)^{2}}$ & $\frac{K}{1+\omega^{2} T^{2}}$ & $-\omega L-2 \arctan \omega T$ \\
\hline SOPTD-2 & $\frac{K e^{-L s}}{\left(T_{1} s+1\right)\left(T_{2} s+1\right)}$ & $\frac{K}{\sqrt{1+\omega^{2} T_{1}^{2}} \sqrt{1+\omega^{2} T_{2}^{2}}}$ & $-\omega L-\arctan \omega T_{1}-\arctan \omega T_{2}$ \\
\hline
\end{tabular}

$A_{\text {osc }}$ and the frequency $\omega_{\text {osc }}$.

(c) As the amplitude $A$ of the oscillation will be not an integer number, calculate the crossing point $C_{o s c}$ using (1). In case of $A$ is an integer number is also possible to use (4).

2. Estimation of steady-state gain by asymmetric oscillations.

(a) Add a bias $d$ to the SSOD block output to produce a stable asymmetric oscillation.

(b) Repeat step 1a.

(c) Once the oscillation is stable, $K$ becomes

$$
K=\frac{\int_{0}^{2 \pi} y(t) d t}{K_{o s c} \int_{0}^{2 \pi} e^{*}(t) d t}
$$

3. Estimate $T$ using data from steps 1 and 2 according to Table 2 .

4. Obtain $L$ using $T$ and the arguments of $C_{i}$ according to Table 2 .

\section{SIMULATION EXAMPLES}

Without loss of generality, the approach can be applied with oscillations of amplitude non-multiple of $\delta$ that means that (1) must be used to calculate $C_{\text {osc }}$ instead of (4). In all the experiments, $\delta$ was set to 1 and the steady-state gain $K$ was obtained by introducing a bias of $\delta=0.5$ in the SSOD block and the approximations were exact.

\subsection{Accuracy of the estimations}

Table III illustrates the actual and process transfer function parameters obtained using information from the oscillations of amplitudes $\delta, 2 \delta$ and $3 \delta$. The frequencies and gains of the current processes represent the points in which the processes intersect with the critical points $C_{1}, C_{2}$ and $C_{3}$ in the Nyquist map. These points are approximations obtained from the $\mathrm{DF}$, whereas the true values are obtained from experimental measures. Note that estimations using $C_{3}$ are very close to the actual processes, and the estimation error in any parameter is below the $2 \%$.

Note that in the presented example of SOPTD two approaches are possible: 1 ) consider that there is a single pole with multiplicity equal to 2 and identify $T$, and 2 ) fix the value of $T_{1}$ and estimate $T_{2}$. The application of the approach to the estimation of two different lags is complicated due to the discrepancies between theoretical and practical critical points, especially in the frequencies, producing imaginary solutions in many situations.

Notice the non-causal model obtained in the last case in Table 3 with the lowest amplitude oscillation due to the discrepancies between the real $\left(\omega_{1}=0.304\right)$ and the theoretical $\left(\omega_{1}=0.404\right)$ oscillations frequency. As the order of oscillation increases, the discrepancy reduces and the estimation parameters converge to the real ones.

\subsection{Comparative with other methods}

Table 4 compares the actual and estimated process transfer functions for first order processes using the event-based and the ATV-2 approaches [8, 10]. Note that the estimations provided by ATV-2 are less accurate. However, since the AVT-2 assumes that the dead time can be observed from the initial response of the system to the relay tests, the delays of the ATV-based results are exact.

\subsection{High-order processes}

Table 4 presents the outcome of the event-based identification method when the process is modeled as FOPTD and SOPTD. With regard to the second case, the results for the SOPTD-1 are not so good due to the dominant lag as the estimation produces an average lag, that is, a critically damped system but, however the real process is overdamped. Applying the SOPTD-2 model when $T_{1}$ is provided, the estimated model can be now considered a good approach to the real one. Nyquist curves of the real processes and estimated transfer functions are presented in Figure 5.

\subsection{Non-minimum phase processes}

In this section, results of the estimation of FOPTD and SOPTD of non-minimum phase processes are presented in Table 6. The second case presented is compared with other method in the literature called phase deviation [15] in Figure 6. Note how this method presents a bad behavior at low frequencies that produces stationary error in contrast to the event-based method proposed in this paper.

\section{DISCUSSION}

In this paper, a simple event-based method to estimate the parameters of first and second order systems with time delays using SSOD sampling has been presented. 
Table 2: Estimation of $T$ and $L$

\begin{tabular}{|c|c|c|}
\hline Model & $\mathbf{T}$ & $\mathbf{L}$ \\
\hline FOPTD & $\frac{\sqrt{K^{2} K_{o s c}^{2}-\left|C_{o s c}\right|^{2}}}{\left|C_{o s c}\right| \omega_{o s c}}$ & $-\frac{\angle C_{o s c}+\arctan \omega_{o s c} T}{\omega_{o s c}}$ \\
\hline SOPTD-1 & $\frac{\sqrt{\left|C_{o s c}\right|\left(K K_{o s c}-\left|C_{o s c}\right|\right)}}{\left|C_{o s c}\right| \omega_{o s c}}$ & $-\frac{\angle C_{o s c}+2 \arctan \omega_{o s c} T}{\omega_{o s c}}$ \\
\hline SOPTD-2 & $T_{1}$ known & \\
& $T_{2}=\frac{\left|C_{o s c}\right|^{2} T_{1} \omega_{o s c}^{2}-K^{2} K_{o s c}^{2}+\left|C_{o s c}\right|^{2}}{\left|C_{o s c}\right|^{2} \omega_{o s c}^{2}\left(T_{1} \omega_{o s c}^{2}+1\right)}$ & $-\frac{\angle C_{o s c}+\arctan \omega_{o s c} T_{1}+\arctan \omega_{o s c} T_{2}}{\omega_{o s c}}$ \\
\hline
\end{tabular}

Table 3: Comparative of the improvement of the estimations using information from three oscillations of different amplitude and frequency.

\begin{tabular}{|c|c|c|c|}
\hline Nominal process & $\mathbf{C}_{\mathbf{1}}$ & $\mathbf{C}_{\mathbf{2}}$ & $\mathbf{C}_{\mathbf{3}}$ \\
\hline$\frac{e^{-s}}{s+1}$ & $\frac{e^{-1.101 s}}{0.782 s+1}$ & $\frac{e^{-0.998 s}}{0.926 s+1}$ & $\frac{e^{-1.006 s}}{0.998 s+1}$ \\
$\omega_{1}=1.39, K_{1}=1.919$ & $\omega_{1}=1.425$ & $\omega_{2}=1.781$ & $\omega_{3}=1.843$ \\
$\omega_{2}=1.76, K_{2}=2.175$ & $K_{1}=1.59$ & $K_{2}=2.03$ & $K_{3}=2.14$ \\
$\omega_{3}=1.89, K_{1}=2.209$ & $A_{1}=1.006$ & $A_{2}=2.009$ & $A_{3}=3.02$ \\
\hline$\frac{e^{-s}}{(s+1)^{2}}$ & $\frac{e^{-0.9965 s}}{(0.930 s+1)^{2}}$ & $\frac{e^{-1.014 s}}{(0.985 s+1)^{2}}$ & $\frac{e^{-1.006 s}}{(0.995 s+1)^{2}}$ \\
$\omega_{1}=0.896, K_{1}=2.007$ & $\omega_{1}=0.963$ & $\omega_{2}=1.127$ & $\omega_{3}=1.185$ \\
$\omega_{2}=1.114, K_{2}=2.432$ & $K_{1}=1.9$ & $K_{2}=2.311$ & $K_{3}=2.451$ \\
$\omega_{3}=1.182, K_{1}=2.523$ & $A_{1}=1.009$ & $A_{2}=2.038$ & $A_{3}=3.037$ \\
& $e^{-1.074 s}$ & $\frac{e^{-1.014 s}}{(s+1)(0.985 s+1)}$ & $\frac{e^{-1.006 s}}{(s+1)(0.991 s+1)}$ \\
\hline
\end{tabular}

Table 4: Parameter estimation of FOPTD with different delays and time constants. The information used in the event-based method corresponds to oscillation of amplitude higher to $4 \delta$.

\begin{tabular}{|c|c|c|}
\hline Process & Event-based & ATV-2 \\
\hline$\frac{e^{-2 s}}{s+1}$ & $\begin{array}{c}\frac{e^{-2.0066 s}}{1.0002 s+1} \\
A=4.565, \omega_{o s c}=1.088 \\
K_{o s c}=1.856\end{array}$ & $\frac{0.89 e^{-2 s}}{0.761 s+1}$ \\
\hline$\frac{e^{-5 s}}{s+1}$ & $\begin{array}{c}\frac{e^{-5.002 s}}{1.008 s+1} \\
A=6.4, \omega_{o s c}=0.5134 \\
K_{o s c}=1.15\end{array}$ & $\frac{0.833 e^{-2 s}}{0.728 s+1}$ \\
\hline$\frac{e^{-2 s}}{10 s+1}$ & $\begin{array}{c}\frac{e^{-2.003 s}}{9.977 s+1} \\
A=5.12, \omega_{o s c}=0.7859 \\
K_{o s c}=8\end{array}$ & $\frac{0.988 e^{-2 s}}{8.02 s+1}$ \\
\hline
\end{tabular}

The method is based on the information that the describing function of the SSOD sampling block provides about the point in which the process starts oscillating when the gain margin with respect to $-1 / N\left(\delta_{A}\right)$ is reduced to zero by applying a proportional gain. The oscillation means the intersection of the process with a point of some arc segment of the SSOD describing function in the Nyquist loci. With the proportional gain applied to reach a stable oscillation, the frequency, the DF information on the intersection point, and the polar forms of the transfer functions of FOPTD and SOPTD, the steady-state gain, the time constants and time delays are obtained.

Comparing the results with other methods based on describing functions analysis of a relay, solutions are
Table 5: Examples of parameter estimation of high-order processes.

\begin{tabular}{|c|c|c|}
\hline Process & FOPTD & SOPTD \\
\hline$\frac{e^{-2 s}}{(2 s+1)^{2}}$ & \multicolumn{2}{|c|}{$A=6.122, K_{o s c}=2.5, \omega_{o s c}=0.623$} \\
\hline$\frac{e^{-2 s}}{(s+1)(10 s+1)}$ & $\begin{array}{l}A=8.164, \\
\frac{e^{-2.869 s}}{11.588 s+1}\end{array}$ & $\begin{array}{c}\tau_{\text {osc }}=6.76, \omega_{\text {osc }}=0.572 \\
\frac{e^{-1.249 s}}{(4.173 s+1)^{2}} \\
\frac{e^{-1.9883 s}}{(1.0197 s+1)(9.9684 s+1)}\end{array}$ \\
\hline$\frac{e^{-0.2 s}}{0.5 s^{2}+1.5 s+1}$ & $\begin{array}{l}A=7.2,1 \\
\frac{e^{-0.460 s}}{2.080 s+1}\end{array}$ & $\begin{array}{c}s c=7.45, \omega_{o s c}=3.514 \\
\frac{e^{-0.1898 s}}{(0.7188 s+1)^{2}}\end{array}$ \\
\hline$\frac{e^{-2 s}}{(2 s+1)^{5}}$ & \multicolumn{2}{|c|}{$\begin{array}{c}A=8.18, K_{\text {osc }}=1.95, \omega_{\text {osc }}=0.2749 \\
\frac{e^{-7.407 s}}{6.025 s+1}\end{array}$} \\
\hline
\end{tabular}

very accurate for non-measurement errors of the limit cycle waveform. The identification accuracy can be indeed high due to the use of oscillations that reduce the high-harmonic components of the limit cycle, especially for identifying higher-order or time delay processes of which the relay responses are apparently different from the sinusoidal shape.

Additionally, as other DF-related methods, the computational cost is low because applications of numerical methods to solve non-linear equations or costly experimental measures of high-order harmonics are not needed.

However, many questions and problems still remain open. One shortcoming of the method is that obtaining high-order oscillations can be sometimes difficult since depends on the historical operation of the pro- 


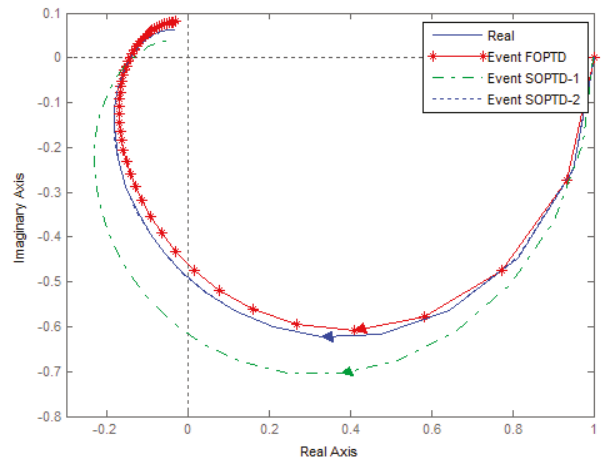

(a)

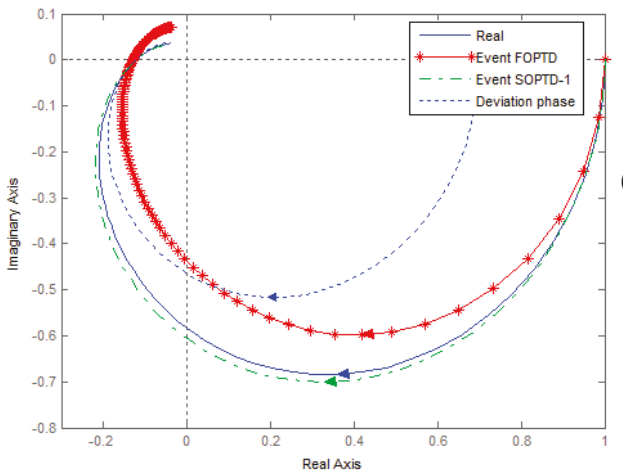

(b)

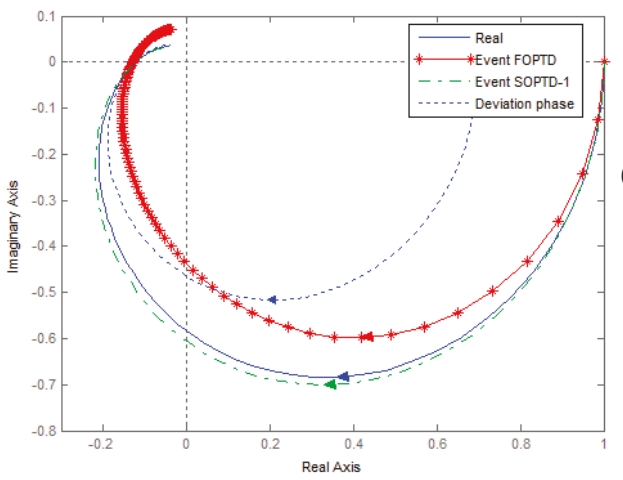

(c)

Figure 5: Nyquist curves of high-order processes and estimated models for three cases presented in Table 5. (a) $\frac{e^{-2 s}}{(s+1)(10 s+1)}$, (b) $\frac{e^{-0.2 s}}{0.5 s^{2}+1.5 s+1}$, (c) $\frac{e^{-2 s}}{(2 s+1)^{5}}$.

cess, not just on the gain. So, a constant increment of the proportional gain to reach higher oscillations can be translated into instability. Other issue to research is that the quantitative relationship between the Cpoint order used and the accuracy of the identification is not known. The study of processes with integrators will also be part of future studies. Furthermore, the sampling technique used for the estimation is vulnerable to the noise of the measurements. How the noise affects the parameter estimation is also an interesting problem to consider in the future.

\section{Agradecimientos}

This work has been funded by Spanish Ministry of Economy and Competitiveness under contracts DPI2012-31303 and DPI2014-55932-C2-2-R.
Table 6: Examples of parameter estimation of high-order processes.

\begin{tabular}{|c|c|c|}
\hline Process & FOPTD & SOPTD \\
\hline$\frac{(1-s) e^{-s}}{(s+1)^{5}}$ & \multicolumn{2}{|c|}{$A=9.46, K_{o s c}=1.5, \omega_{o s c}=0.4648$} \\
\hline$\frac{(1-s) e^{-4 s}}{(s+1)^{4}}$ & \multicolumn{2}{|c|}{$\begin{array}{c}A=7.09, K_{o s c}=1.19, \omega_{o s c}=0.3459 \\
\frac{e^{-7.20 s}}{1.813 s+1} \\
\frac{e^{-6.499 s}}{(1.228 s+1)^{2}}\end{array}$} \\
\hline
\end{tabular}

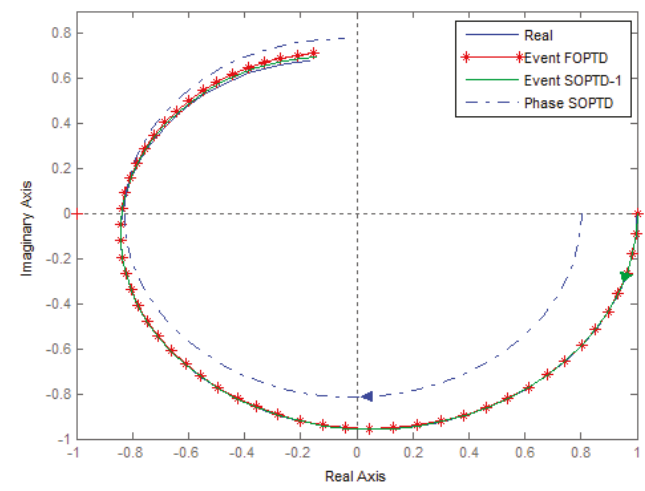

(a)

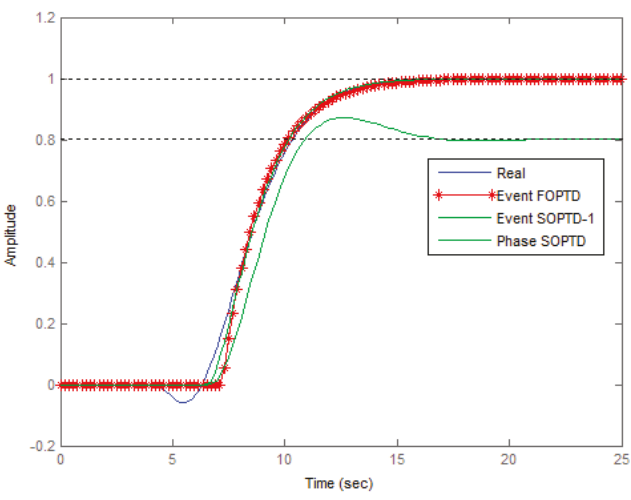

(b)

Figure 6: (a) Nyquist plots and (b) step responses for the second case in Table 6 . The event-based method is compared with the strategy proposed in [15].

\section{Referencias}

[1] K.E. Årzén, A Simple Event-Based PID Controller, 14th IFAC World Congress (1999), Beijing, China.

[2] B. Bernhardsson, K.J. Åström, Comparison of periodic and event based sampling for first-order stochastic systems, 14th IFAC World Congress (1999), Beijing, China.

[3] M. Beschi, S. Dormido, J. Sanchez, A. Visioli, Characterization of symmetric send-on-delta PI controllers, Journal of Process Control 22(10) (2012) 1930-1945.

[4] M. Beschi, S. Dormido, J. Sanchez, A. Visioli, L.J. Yebra, Event-based PI plus feedforward control strategies for a distributed solar collector field, IEEE Transactions on Control Systems Technology 22(4) (2014) 1615-1622. 
[5] M. Beschi, S. Dormido, J. Sánchez, A. Visioli, Closed-loop automatic tuning technique for an event-based PI controller, Industrial \& Engineering Chemistry Research 54(24) (2015) 6362-6370.

[6] S. Dormido, M. Mellado, Determination of limit cycles in adaptive sampling systems (In Spanish), Revista de Automática 26 (1975) 21-31.

[7] S. Dormido, M. Mellado, A study on fixeddifference sampling scheme, Applications and Research in Information Systems and Sciences 2 (1977) 496-500.

[8] I. Kaya, D.P. Atherton, Parameter estimation from relay autotuning with asymmetric limit cycle data, Journal of Process Control 11(4) (2001) 429-439.

[9] E. Kofman, J. Braslavsky, Level crossing sampling in feedback stabilization under data rate constraints, 45th IEEE International Conference on Decision and Control (2006), San Diego, USA.

[10] W. Li, E. Eskinat, W.L. Luyben, An improved autotune identification method, Industrial \& Engineering Chemistry Research 30(7) (1991) 15301541.

[11] M. Miskowicz (ed.), Event-Based Control and Signal Processing, CRC Press, 2015.

[12] M. Miskowicz, Send-on-delta: An event-based data reporting strategy, Sensors 6 (2006) 49-63.

[13] C. Scali, G. Marchetti, D. Semino, Relay with additional delay for identification and autotuning of completely unknown processes, Industrial \& Engineering Chemistry Research 38(5) (1999) 1987-1997.

[14] V. Vasyutynskyy, K. Kabitzsh, Implementation of PID controller with send-on- delta sampling, International Control Conference (2006), Glasgow, Scotland, United Kingdom.

[15] P. Wang, D. Gu, W. Zhang, Modified relay feedback identification based on describing function analysis, Industrial \& Engineering Chemistry Research 46(5) (2007) 1538-1546. 